\title{
Le fait viti-vinicole en Chine dans le champ des sciences humaines et sociales. Premiers résultats et programme de recherche
}

\author{
Guillaume Giroir \\ Université d'Orléans, Département de Géographie, 10 rue de Tours, 45065 Orléans, France
}

\begin{abstract}
Résumé. Depuis les années 2000, la Chine a connu une véritable « fièvre du vin », avec un fort développement de la production et de la consommation, ainsi que de l'oenotourisme. Néanmoins, le fait viti-vinicole chinois, en tant qu'objet scientifique global, reste encore très mal connu dans le champ des sciences humaines et sociales. Si les travaux des chercheurs chinois (Li Hua, Yang Hecai, Li Jiagui, Lu Zhi, Li Demei ... ) se développent, les recherches occidentales sont quasiment inexistantes (G. Giroir). L'objectif de cette étude, d'inspiration géographique mais résolument pluridisciplinaire, est donc de proposer divers types de méthodologies, de modèles théoriques, d'analyses empiriques et d'approche quantitative pour essayer de décrire et comprendre la complexité des réalités vitivinicoles chinoises. Elle présente les premiers résultats obtenus par G. Giroir dans ce nouveau champ de recherche, tout en proposant un programme de recherches. L'analyse présente successivement : un état des lieux de la recherche chinoise et occidentale ; divers modèles et théories ; les modalités possibles d'une 《archéologie » (M. Foucault) du vignoble chinois d'inspiration systémique et multiscalaire ; des études de cas de 《châteaux viti-vinicoles 》; la présentation d'une base de données multicritère sur la totalité des entreprises viti-vinicoles en Chine (environ 900).
\end{abstract}

\section{Introduction}

La Chine s'est longtemps présentée comme le leader des pays du Sud et constitue le cas le plus spectaculaire d'émergence d'un grand pays autrefois pauvre (re)devenu une puissance économique majeure. Depuis les années 2000, la Chine a connu une véritable « fièvre du vin », avec un fort développement de la production et de la consommation, ainsi que de l'oenotourisme. Néanmoins, le fait viti-vinicole chinois reste encore très mal connu dans le champ des sciences humaines et sociales. Si les travaux des chercheurs chinois (Li Hua, Yang Hecai, Li Jiagui, Lu Zhi, Li Demei...) se développent, les recherches occidentales sont quasiment inexistantes (G. Giroir).

À l'évidence, l'étude du fait viti-vinicole (ou « viticolité ») en Chine en tant qu'objet scientifique global représente un vaste champ de recherche potentiel. En revanche, la question reste entière de définir les outils méthodologiques, théoriques et interprétatifs les plus adéquats pour explorer ce phénomène. En effet, le caractère récent du fait viti-vinicole moderne en Chine, le gigantisme du territoire, l'altérité culturelle, le nombre élevé des entreprises viti-vinicoles, les discontinuités et fractures de l'histoire chinoise contemporaine constituent autant de spécificités et de défis auxquels le discours et la pratique scientifiques doivent s'adapter.

L'objectif de cette étude, inscrite dans le champ de la géographie mais aussi résolument pluridisciplinaire, est donc de proposer divers types d'approches méthodologiques et de modèles théoriques pour essayer de saisir la complexité des réalités vitivinicoles chinoises. Elle présente également les premiers résultats obtenus par G. Giroir dans ce nouveau champ de recherche. Elle offre à la fois un bilan et un programme de recherches du fait viti-vinicole en Chine. Ainsi, une première partie théorique présentera les divers modèles susceptibles de permettre l'interprétation du fait vitivinicole en Chine : théories diffusionnistes (transposition de la culture du vin d'une civilisation à une autre) ; théories néo-libérales (convergence universelle des modes de consommation grâce au marché) ; théories géopolitiques et géoculturelles fondées sur le modèle Centre/Périphérie (triomphe de la civilisation occidentale sur les alcools blancs traditionnels, et inversement fin de l'hégémonie viti-vinicole européenne, ou métissage des civilisations conçues comme des « mosaïques »?) ; théories systémiques (modalités d'articulation entre cette greffe culturellement allogène et la civilisation chinoise, Giroir, 2002) [1].

Dans une deuxième partie, on insistera sur le rôle majeur d'une approche historique renouvelée en introduisant une double dimension multiscalaire et systémique. Il s'agira à la fois de reconstituer la logique et la cohérence des évolutions à échelle macro, celle de la Chine toute entière, mais aussi d'identifier à une échelle micro-territoriale la trajectoire particulière des entreprises et des cépages. Elle présentera par exemple les travaux de G. Giroir sur l'entreprise Changyu. On évoquera la nécessité de procéder à un travail de dépouillement des archives des missionnaires catholiques à l'origine de nombreuses entreprises viti-vinicoles chinoises. Il s'agira également de montrer la diffusion des grands cépages internationaux en Chine mais aussi d'identifier l'histoire singulière de certains cépages d'origine française disparus du fait du phylloxéra, mais conservés en Chine.

Une troisième partie, à échelle micro-territoriale, proposera une approche de type empirique fondée sur des études de cas. Elle présentera les premiers résultats des 
enquêtes de terrain de G. Giroir dans certaines entreprises viti-vinicoles de Pékin, Tianjin, du Hebei (Changli) et du Shandong (Yantai), comme par exemple la lecture géosémiotique du parc à thème sur le vin de Château Changyu AFIP Global près de Pékin.

Dans une dernière partie quantitative et cartographique seront présentés les premiers résultats de l'élaboration d'une lourde base de données multicritère concernant un millier d'entreprises viti-vinicoles chinoises.

\section{Le fait viti-vinicole en Chine dans le champ des sciences sociales : état des lieux de la recherche, problématique et modèles théoriques}

Le sujet abordé ici ne porte pas seulement sur le vin en tant que produit de consommation, ni même seulement sur les domaines viticoles en tant qu'espaces productifs ou touristiques. Il traite de la 《viticolité ». L'approche retenue s'inspire des approches systémiques classiques mais en leur donnant une acception la plus large possible ${ }^{1}$. Objet scientifique total, le système viti-vinicole désigne ainsi un ensemble de terroirs, de techniques agronomiques, de paysages, de formes d'habitat, d'acteurs (vignerons, entreprises, organes professionnels, autorités politiques, distributeurs...), de marchés, de médias, de valeurs, de symboles et d'images. Infiniment différencié et hiérarchisé, la vigne et le vin sont aussi appréhendés comme facteurs de développement local et de marketing territorial. Patrimoine paysager, social et culturel, ils contribuent à construire l'identité mais aussi la qualité des territoires. Plante de civilisation, la vigne est également civilisatrice au sens le plus large.

\subsection{Un champ de recherche encore émergent}

Après la longue interruption de la période maoïste, l'essor véritable du fait viti-vinicole en Chine est très récent et les recherches encore rares dans le domaine des sciences humaines et sociales.

\subsection{1. État des lieux des recherches sur la vigne et le vin en Chine}

Le premier ouvrage œnologique chinois après 1949 a été publié par Zhu Mei en 1954 [2]. Le nombre des publications sur le vin en Chine s'est élevé à 10583 pour la période 1986-2012, mais dans la première décennie

\footnotetext{
${ }^{1}$ Parmi les approches systémiques appliquées aux territoires vitivinicoles français, on peut citer : F. Auriac (1979) Système économique et espace (un exemple en Languedoc), thèse de doctorat ; publiée en 1983 sous le titre Système économique et espace, Economica ; et J. Maby (1995), La Trame du vignoble, géographie d'une réussite viticole en vallée du Rhône, éd. A. Barthélémy, Avignon ; (2002), Campagnes de recherche, approche systémique de l'espace rural, Habilitation à diriger des recherches, UMR Espace Université d'Avignon ; (2007), 《Systèmes spatiaux viticoles. Trois cas 》, site web Géoconfluences, École Normale Supérieure de Lyon, 13 p. Voir aussi J.-C. Hinnewinkel (2004) Les Terroirs Viticoles. Origines et devenirs, Éditions Féret.
}



Figure 1. Évolution du nombre de publications sur le vin en Chine (1986-2012). Source : www. wanfangdata.com.cn/ banques de données universitaires chinoises (consultées le 22 juillet 2013).

jusqu'en 1997, seules 22 études ont été publiées ${ }^{2}$. Les premières recherches véritables sur le vin en Chine remontent à 1998, où 39 publications ont vu le jour. Cette année-là, Gu Qichang a publié Cinquante ans d'histoire $d u$ vin de la Chine nouvelle [3]. Il y a retracé l'histoire de la culture de la vigne et l'évolution de l'œnologie, puis dressé un état des lieux du vin 50 ans après la fondation de la Chine populaire, et donné des aperçus sur les perspectives de développement de l'industrie viti-vinicole en Chine.

L'année 2001, date de l'entrée de la Chine dans l'OMC, marque le véritable décollage des recherches en matière vitivinicole. Après 2011, on recense en Chine plus de mille publications sur le vin chaque année. Au cours de ces trente dernières années, les recherches ont surtout porté sur : la culture des vignes (cépage, pédologie, techniques de culture, météorologie), les techniques de fabrication du vin (odeur, qualité, chimie, agro-alimentaire), le génie biologique du vin (régénération des cépages occidentaux importés) et les stratégies marketing du vin (comparaison vin étranger/chinois, production et vente du vin dans les pays occidentaux, développement des entreprises viticoles, marché du vin).

Dans le domaine des sciences humaines et sociales, les recherches sur le vin en Chine privilégient l'économie. Elles sont souvent menées par des chercheurs des instituts agro-alimentaires ou des économistes rattachés à une entreprise viti-vinicole, notamment Changyu. Les recherches portant sur la dimension territoriale du vin sont très rares et concernent surtout : les indications géographiques des vins ; la division régionale des territoires viticoles (selon les entités administratives, les climats, les sols ou les cépages); les stratégies de développement des entreprises viticoles et l'œnotourisme. Parmi les 10583 publications de la période 1986-2012, seulement 5 l'ont été dans des revues de géographie.

La question des indications géographiques des vins a été abordée par Li Hua dans les années 1990. Inspiré par l'expérience française, il a proposé d'établir un système complet d'indications géographiques pour la production de vin chinois. Tang Wenlong a également effectué des recherches sur ce sujet. Yang Hecai a lancé une série de recherches pour contribuer à faire émerger ce secteur agroindustriel à fort potentiel en Chine : nouveau découpage

\footnotetext{
${ }^{2}$ Il s'agit des publications (articles de revue, thèses, actes de colloques) dont le titre inclut le mot clé «vin» (putaojiu).
} 
Tableau 1. Principaux chercheurs chinois dans le domaine du vin.

\begin{tabular}{|c|c|}
\hline Nom, rattachement institutionnel & $\begin{array}{l}\text { Domaines de recherche sur le vin (nombre de } \\
\text { publications) }\end{array}$ \\
\hline $\begin{array}{l}\text { DUAN Changqing (段长青), 中国农业大学 中国 } \\
\text { 农业大学食品科学与营 养工程学院葡萄与匍萄 } \\
\text { 酒研究中心主任 中国园 艺学会葡萄与葡萄酒分 } \\
\text { 会秘书长. Directeur du Centre de recherches sur la } \\
\text { vigne et le vin de l'Université agricole de Chine. } \\
\text { Premier secrétaire de la sous-commission de la vigne } \\
\text { et du vin (Association horticole de Chine). }\end{array}$ & génie biologique ; culture de différents cépages (40) \\
\hline $\begin{array}{l}\text { LI Demei (李德美), 北京 农学 Université agricole de } \\
\text { Pékin }\end{array}$ & $\begin{array}{l}\text { œnologie (notamment échanges France/Chine); stratégies de } \\
\text { développement de l'industrie du vin (2005); Pékin et } \\
\text { Hebei (12) }\end{array}$ \\
\hline $\begin{array}{l}\text { LI Hua (李华), 西北农林 科技大学葡萄酒学院 } \\
\text { Professeur à l'Université des sciences et des technolo- } \\
\text { gies agricoles et sylvicoles de la Chine du Nord-Ouest. }\end{array}$ & $\begin{array}{l}\text { indications géographiques et leur protection en Chine ; } \\
\text { division territoriale viticole (selon le climat); types de } \\
\text { cépages ; types d'exploitations (châteaux, clos, champs) (64) }\end{array}$ \\
\hline $\begin{array}{l}\text { LI Jiagui (李甲贵), 西北 农林科技大学 Professeur à } \\
\text { l'Université des sciences et technologies agricoles et } \\
\text { sylvicoles du Nord-Ouest. }\end{array}$ & $\begin{array}{l}\text { culture du vin (putao wenhua) en Chine ; marketing du vin ; } \\
\text { vin de luxe ; didactique du vin ; tourisme viticole (2008) (22) }\end{array}$ \\
\hline $\begin{array}{l}\text { LI Jiming (李记明), 张裕 集 团公司 Cadre de } \\
\text { l'entreprise Changyu }\end{array}$ & $\begin{array}{l}\text { œnologie (dont vin de glace et brandy); histoire récente de } \\
\text { la culture du vin en Chine (2009); vin français. }\end{array}$ \\
\hline $\begin{array}{l}\text { LIU Shisong (刘 世松), 烟台市葡萄与葡萄酒局, } \\
\text { Bureau de la vigne et du vin de Yantai }\end{array}$ & châteaux viticoles à Yantai (2011). \\
\hline $\begin{array}{l}\text { LU Huilin (鲁会玲), 黑, 龙 江 省农业 科 学 院 } \\
\text { Professeur de l'Institut agricole du Heilongjiang }\end{array}$ & $\begin{array}{l}\text { techniques vinicoles ; faisabilité de nouveaux vignobles dans } \\
\text { la zone froide du Heilongjiang (16) }\end{array}$ \\
\hline MA Huiqin (马 会 勤), Université agricole de Chine & $\begin{array}{l}\text { génie biologique ; marché de consommation à Pékin (2007) } \\
(42)\end{array}$ \\
\hline $\begin{array}{l}\text { TANG Wenlong (唐文龙), 山东工商学院, 烟台 } \\
\text { 张裕集团有限公司 Professeur à l'Institut de } \\
\text { l'Industrie et du Commerce du Shandong et à } \\
\text { l'entreprise Changyu }\end{array}$ & $\begin{array}{l}\text { marketing ; marché du vin (2006) ; stratégie des entreprises ; } \\
\text { indications géographiques; rédaction de rapports annuels sur } \\
\text { le marché chinois (78) }\end{array}$ \\
\hline $\begin{array}{l}\text { YANG Hecai (杨和才), 西北农林科技大学 } \\
\text { Professeur à l'Université des sciences et technologies } \\
\text { agricoles et sylvicoles du Nord-Ouest }\end{array}$ & $\begin{array}{l}\text { didactique ; culture du vin en Chine ; marketing ; } \\
\text { régionalisation du vin (2008); normes de production en } \\
\text { Chine ; cépages ; indications géographiques en Chine (2008) } \\
(27)\end{array}$ \\
\hline
\end{tabular}

régional des territoires viticoles, institutionnalisation du marché de production et de vente du vin, stratégie de développement territorial $\mathrm{du}$ vin. Les recherches sur l'œnotourisme sont très récentes en Chine. Seules 59 études ont été publiées ces dernières années. Li Jiagui a travaillé sur le potentiel de développement oenotouristique à Weiwu (Gansu) et Penglai (Shandong) dès 2008. L'essor de l'œnotourisme par le biais de châteaux dans la péninsule du Shandong commence à être étudié. Certaines recherches portent sur les conflits d'utilisation du sol induits par l'essor de la viti-viniculture : ainsi, le grand spécialiste des pandas, Lu Zhi (Beijing University) a montré les risques de conflit entre nouveaux vignobles et aires protégées dédiées aux pandas [4].

\subsubsection{Recherches occidentales sur les vignobles chinois}

Encore plus rares sont les chercheurs occidentaux traitant des territoires viticoles chinois ${ }^{3}$. Dès 1985, l'œnologue

\footnotetext{
${ }^{3}$ Certains chercheurs ont exploré d'autres vignobles asiatiques : ex. J.-R. Pitte (1983) 《Vignobles et vins du Japon », Annales de Géographie, $\mathrm{N}^{\circ}$ 510, mars-avril, p. 172-199, ou G. Banks et alii (2013) «Wines without Latitude : Global and local forces
}

languedocien D. Boubals avait publié un article sur l'essor de la viti-viniculture en Chine [5], puis A. Löwenstein en 1991 [6]. En 2002, G. Giroir a fait une communication et un article sur la question viticole en Chine en montrant l'articulation entre civilisation et mondialisation. À la suite d'enquêtes de terrain en octobre et décembre 2012 (Pékin/Hebei/Tianjin/Shandong), il a commencé à participer à des conférences internationales et à publier une série de travaux de terrain sur ce sujet ${ }^{4}$.

\subsection{Problématique et types d'approches théoriques}

\subsubsection{Problématiques}

La problématique centrale est celle de la transition, ou des transitions. Il ne s'agit pas seulement du processus complexe de transition entre économie planifiée et

and the geography of the Thai wine industry », EchoGéo, 23, janvier/mars.

${ }^{4}$ À noter l'utile Putao niangliu zhiqiao (Le pont de l'œnologie. Dictionnaire æenologique), (2012) de Carlo Queruli, Colombin e Figlio Spa, Chine (multilingue : chinois/ italien/français/anglais/allemand). 
économie de marché, mais d'une véritable « transition de civilisation ${ }^{5} \gg$ pour une Chine plus urbaine, mobile et globalisée.

$\mathrm{Au}$ sein de cette macro-transition, quelles sont les modalités de passage du baijiu, alcool blanc emblématique de la civilisation chinoise, au vin de raisin, symbole de la civilisation occidentale ? Quelles sont les modalités de construction de ce nouveau système viticole ? Ces recherches comportent une dimension expérimentale : il s'agit d'approfondir la notion d'émergence d'un système territorial (en l'occurrence viticole). L'une des principales questions tient aussi à la spécificité des territoires vitivinicoles émergents en Chine. S'inscrivent-ils dans le dualisme entre les « modèles viticoles » de l'Ancien Monde (France, Italie, Espagne....) et ceux du Nouveau Monde (États-Unis, Australie, Nouvelle-Zélande, Chili, Argentine...), ou bien inaugurent-t-ils un type de système viticole inédit, une combinatoire originale empruntant aux deux macro-entités citées, tout en s'en différenciant fondamentalement?

À un niveau plus analytique, on peut se demander quel est le contenu de la viticolité chinoise ${ }^{6}$. Cette « fièvre du vin » aboutit-elle à un système viticole a minima, à de simples espaces productifs et touristiques régis par la seule logique d'entreprise et de profit, ou à la mise en place d'une véritable civilisation du vin de type européen (société de vignerons) ? Comment s'articule cette greffe culturelle allogène avec la civilisation chinoise plurimillénaire ? La viticolité occidentale, et notamment européenne (terroir ; architecture ; oenotourisme ; pratiques œnologiques ; modes de boire...) est-elle transposée telle quelle, ou bien modifiée, réinterprétée, voire altérée ? Quelle peut être la signification de la vitivinicolité, étroitement liée à la religion chrétienne, dans une civilisation non-chrétienne?

\subsubsection{Types d'approche théorique}

L'émergence de territoires viticoles en Chine s'apparente en partie à la transposition d'un objet/concept (la viticolité) d'un macro-territoire (ici l'Europe) à un autre (la Chine) ; ce mécanisme n'est pas sans analogie, mutatis mutandis, avec la circulation d'autres modèles (architecturaux, urbains, juridiques...). Mais selon quels modèles heuristiques ou théories interpréter ce transfert ?

Il est possible d'engager une première approche morpho-fonctionnelle. Selon elle, ce transfert relève du champ des théories diffusionnistes associées aux théories de l'information et de la dynamique des systèmes. Il s'applique aux biens matériels et aux idées, religions, technologies, langues ou images. En tant que système global, à la fois matériel et idéel ${ }^{7}$, la viticolité se

\footnotetext{
${ }^{5}$ G. Giroir (1994) Transition et territoire en Chine. Le cas des périphéries de Pékin, Habilitation à diriger des recherches, U. d'Orléans, $405 \mathrm{p}$.

${ }^{6}$ Encore au début des années 2000, je pouvais conclure à l' 《 absence de système territorial vinicole en Chine » ou affirmer à bon droit 《Le tourisme vinicole reste inexistant 》: G. Giroir (2002), op. cit., p. 273 et 275.

${ }^{7}$ Distinction approfondie par l'anthropologue M. Godelier (1989) L'idéel et le matériel. Pensée, économies, sociétés, Fayard, Paris.
}

Tableau 2. Communications et publications de G. Giroir sur les vignobles chinois.

\begin{tabular}{|c|c|}
\hline 2001 & $\begin{array}{l}\text { 《Vin, mondialisation et civilisation en Chine », } \\
\text { communication publiée dans Douro, actes du } \\
\text { symposium de Porto (Portugal) 《La vigne et le } \\
\text { vin dans le monde », Université de Porto } \\
\text { (Portugal), septembre 2002, vol. 13, p. 255-283. }\end{array}$ \\
\hline 2013 & $\begin{array}{l}\text { "The wine châteaux in China: the case of Yantai } \\
\text { (Shandong province) », communication à } \\
\text { l'Annual Meeting of the Association of American } \\
\text { Geographers (AAG), Los Angeles (États-Unis) }\end{array}$ \\
\hline 2013 & $\begin{array}{l}\text { 《I castelli viticoli in Cina : il caso di Yantai », } \\
\text { Comité national des géographes français, } \\
\text { Commission de géographie rurale, Florence } \\
\text { (Palais Médicis-Riccardi, Italie) (communication } \\
\text { en italien) (article soumis) }\end{array}$ \\
\hline 2013 & $\begin{array}{l}\text { «L'entreprise Changyu, acteur majeur de la } \\
\text { construction du système viticole chinois », } \\
\text { communication au colloque Unesco de } \\
\text { Florianopolis (Brésil) à paraître en } 2014 \text { dans } \\
\text { Cultur. Revista de Cultura e Turismo, Ilheus- } \\
\text { Bahia (Brésil), Universitade Estadual de Santa Cruz }\end{array}$ \\
\hline 2014 & $\begin{array}{l}\text { « The wine theme park Château Changyu AFIP } \\
\text { Global (Beijing) : a geo-semiotic approach », } \\
\text { communication à l'Annual Meeting of the } \\
\text { Association of American Geographers (AAG ; } \\
\text { Wine speciality Group), Tampa (États-Unis); } \\
\text { (article soumis) }\end{array}$ \\
\hline 2015 & $\begin{array}{l}\text { « Le marché du vin en Chine : entre ivresse et } \\
\text { réalités », in François Legouy (coord.) Atlas sur } \\
\text { la vigne et le vin dans la mondialisation, } 2 \text { p., A. } \\
\text { Colin (à paraître) }\end{array}$ \\
\hline
\end{tabular}

prête parfaitement à ce genre d'approche ${ }^{8}$. Dans les années 1960, apparaissent les théories de diffusion de l'innovation ${ }^{9}$. Les théories de la communication ajoutent l'analyse des relais/vecteurs de diffusion entre foyer émetteur et espace récepteur. Divers mécanismes peuvent être identifiés : ils peuvent faire l'objet de modélisation (stades) et de cartographie (aires).

En effet, l'émergence de territoires viticoles est structurée par un puissant système d' « acteurs » qui en assurent la diffusion : communauté d'expatriés ; vignerons (investisseurs privés et cultivateurs) ; « experts nomades » (notamment les œnologues) ; entreprises viticoles ; fonds d'investissements ; distributeurs ; foires ; institutions politiques (du gouvernement central aux gouvernements locaux) ; médias et réseaux sociaux ; autorités politiques ; classes sociales (notamment les élites) ; écoles de formation... Il y a là un vaste champ d'investigation à explorer. Les modalités de la réception sont variables : s'agit-il de l'importation telle quelle de l'héritage étranger (mimétisme) ou d'une transformation (adaptation, acculturation), voire d'une assimilation ? Cette approche

\footnotetext{
${ }^{8}$ L'exportation de cépages européens en Chine s'inscrit dans le champ des recherches ethno-botaniques (cf. A. von Humboldt à la fin du XIXème).

${ }^{9}$ E.M. Rogers (1962). Diffusion of Innovations. Glencoe : Free Press. T. Hagerstrand (1967) Innovation diffusion as a spatial process. Traduit par A. Pred., University of Chicago Press.
} 
comprend aussi l'évaluation de l'impact des apports étrangers (substitution/diversification).

Une deuxième approche peut être d'ordre géoéconomique ou géopolitique. Le vin constitue aussi un produit de consommation et donc un marché. Son émergence en Chine peut offrir trois lectures contradictoires. La première, d'inspiration néo-libérale, considère le transfert de la culture européenne du vin en Chine comme l'une des multiples manifestations des théories de la transition-globalisation (triomphe de l'économie de marché ; convergence universelle des modes de consommation $)^{10}$. L'essor du marché du vin en Chine représente aussi l'une des grandes phases historiques d'avancée de la mondialisation de cette plante miracle par le biais des Européens. Une deuxième lecture issue des théories néo-marxistes de type Centre/Périphérie est possible. Nombre d'auteurs ont dénoncé les multiples formes de domination de l'Occident ${ }^{11}$. La Chine abonde en héritages architecturaux de la période de semicolonisation $^{12}$. Une lecture possible du transfert du modèle viti-vinicole européen en Chine est donc celle du néoimpérialisme (importation de matériel biologique : ex. cépages) ; dépendance à l'égard d'experts œnologues ; importation d'équipements : tonneaux français, cuves italiennes ...; formes architecturales allogènes). La guerre commerciale entre les importations de vin étranger et vins chinois locaux s'inscrit dans cette problématique. Une troisième lecture, inverse, est celle où la Chine, en s'appropriant et en développant l'un des éléments les plus symboliques de l'occidentalité, prend sa revanche sur l'Occident, le prive de l'un de ses privilèges ; selon une relation maître/disciple, la Chine chercherait à dépasser l'Occident, même dans ce qu'il a de plus emblématique en matière de supériorité culturelle.

L'approche géoculturelle, en partie liée à la précédente, offre un troisième type d'approche possible. Au sein de ce champ scientifique très vaste, l'anthropologie culturelle et la géographie culturelle s'attachent à conceptualiser la diffusion trans-culturelle ${ }^{13}$. La question de la civilisation est au cœur de ce champ de recherche car la vigne (à vin), beaucoup plus qu'un produit alimentaire, incarne aussi le symbole d'une civilisation, une plante de civilisation ainsi qu'une plante civilisatrice. La géographie du boire obéit à des facteurs géographiques ou techniques, mais

\footnotetext{
${ }^{10}$ Sur la mondialisation des aliments : cf. les anthropologues L. Plotnicov, R. Scaglion (eds.) (1999) The Globalization of Food, Waveland.

${ }^{11}$ Parmi eux : S. Latouche (1989) L'Occidentalisation du monde : Essai sur la signification, la portée et les limites de l'uniformisation planétaire, Paris, La Découverte ; E. Saïd (1978) Orientalism, Pantheon Books ; (1993) Culture and Imperialism, Knopf.

12 Wong R. (2007), « Preservation of Traditional Buildings with Heritage Value in Asian Cities with Colonial BackgroundShanghai, Guangzhou and Hong Kong Cases », Housing Science, no. 4, Vol. 31,

${ }^{13}$ Cf. les travaux pionniers d'A. L. Kroeber (1940) « Stimulus Diffusion », American Anthropologist, New Series, Vol. 42, No. 1, Jan./Mar., p. 1-20.
}

aussi et surtout culturels ${ }^{14}$. La question culturelle se pose d'abord sous la forme de la transition d'un mode de boire à un autre. Traditionnellement, la Chine est le pays des alcools de grains distillés ${ }^{15}$ (sorgho, riz, millet...) ou baijiu (littéralement « alcool blanc »). Quand il est question d'alcool en Chine sans autre précision, il s'agit toujours de baijiu (ou parfois de shaojiu), donc d'alcool blanc ${ }^{16}$. Pour parler de vin, la langue chinoise spécifie 《vin de raisin » (putao jiu) (ou même tout simplement hongjiu, vin rouge). Ces alcools forts (le Maotai ou «Moutai », alcool distillé à base de sorgho fermenté titrant $53^{\circ} \mathrm{C}$ ) tiennent une place éminente dans la culture et la société chinoises. $\mathrm{Ne}$ dit-on pas que le fameux poète $\mathrm{Li}$ Bai s'est noyé en voulant trinquer avec la lune ? Les penseurs taoïstes prétendaient qu'il convenait de boire quelques coupes d'alcool blanc pour être accordé au Grand Processus $(\mathrm{TaO})^{17}$. Toutefois, il n'y a pas à proprement parler de géographie des baijiu, mais plutôt seulement une distribution spatiale sélective des entreprises de distillation (Moutai dans la province du Guizhou, Wuliangye dans celle du Sichuan...). Cette distribution n'a que très peu à voir avec les conditions physiques des territoires, leur topographie, leurs sols, leur climat. Le baijiu ne crée pas non plus de paysages agraires ni de villages ou de sociétés spécifiques. Les champs de grains sont indifféremment utilisés pour la consommation alimentaire et la fabrication de l'alcool. Si les méthodes de distillation, mais aussi l'âge font varier considérablement la qualité et le prix final desbaijiu, la notion de terroir s'avère ici peu opératoire, voire non pertinente. L'approche culturelle se présente ensuite par rapport à la transplantation d'un modèle allogène. Dans le domaine du vin, le processus de transplantation dans d'autres aires culturelles interroge la notion même de civilisation ainsi que les relations inter-culturelles. Ce champ théorique est balisé par un double débat. Le premier oppose la vision essentialiste (les civilisations comme entités homogènes et invariantes) et la vision diffusionniste (importance des apports étrangers). Le second porte sur la nature des relations entre civilisations, avec notamment l'opposition entre conflit et $\operatorname{choc}^{18}$ ou, au contraire, convergence,

\footnotetext{
${ }^{14}$ Sur les relations boissons/civilisations : X. De Planhol (1995) L'eau de neige. Le tiède et le frais. Histoire et géographie des boissons fraîches. Paris, Fayard. B. Kiellgren (2004) « Drunken modernity : wine in China », Anthropology of food, 3, december, p. $1-17$.

15 À la différence du saké japonais, qui est fermenté : N. Baumert (2011) Le saké, une exception japonaise, Presses universitaires de Rennes/Presses universitaires François Rabelais ; préface de J.-R. Pitte.

${ }^{16}$ Pour le caractère « jiu 》, le Dictionnaire Ricci (1986) donne les sens suivants : « boisson fermentée ; boisson alcoolique ; vin ; liqueur 》 (p. 191).

${ }^{17}$ Sur les vertus de l'ivresse en Chine : J. Pimpaneau (2000) Célébration de l'ivresse, éd. P. Picquier.

${ }^{18}$ S. Huntington (1993) « The Clash of Civilizations, », Foreign Affairs, Vol. 72, no. 3, Summer, p. 22-49 ; (1996) The Clash of Civilizations and the Remaking of World Order, New York, Simon \& Schuster.
} 
complémentarité, partage, métissage ${ }^{19}$. Ce double débat trouve une forte résonnance en Chine. Bien des formes d' « asiatisation/sinisation du monde » existent ${ }^{20}$. Mais, l'émergence de territoires viticoles en Chine pose la question inverse, celle de l' « occidentalisation de la civilisation chinoise $»$. Si l'exceptionnelle continuité de la civilisation chinoise depuis plus de quatre mille ans peut susciter des discours essentialistes sur une Chine totalement différente, éternelle, immuable et homogène, elle apparaît aussi comme une « civilisation-mosaïque » qui a su s'enrichir d'éléments matériels et immatériels allogènes, occidentaux ou non ${ }^{21}$. Les territoires viticoles émergents comportent donc des enjeux multiples et essentiels interrogeant la civilisation chinoise elle-même.

Le troisième aspect de l'approche culturelle est celui de la « culture du vin »(putaojiu wenhua). L'étude de G. Giroir de 2002 [1] aborde certains traits de cette question majeure. Elle se demande si la « fièvre du vin » est l'expression de la mondialisation culturelle. Elle s'interroge sur les limites de la portée socio-culturelle mais aussi territoriale de cet engouement pour le vin, en insistant sur la notion de « filtrage civilisationnel ». « Le vin ne présente aucune cohérence avec la civilisation chinoise ». La consommation per capita y est encore très faible, et il n'y a pas encore de véritable « système territorial vinicole en Chine » (rythme climatique de la vigne en porte-à-faux avec la mousson estivale, absence de société vigneronne, d'habitat vigneron, quasi-inexistence des notions de terroir et de qualité, absence de vins de garde, rareté de l'oenotourisme). Elle décrit les phénomènes et processus interdisant toute transposition mimétique en Chine de la civilisation européenne de la vigne et du vin. Elle aborde également la question des modes de consommation du vin en Chine. Le vin souffre encore d'une infériorité historique et culturelle par rapport aux baijiu, notamment au Maotai, véritable alcool national célébré par les poètes

\footnotetext{
${ }^{19}$ Cf. les notions de « civilisation de l'Universel », de 《 civilisation du vivre-ensemble 》 (par le théologien-naturaliste P. Teilhard De Chardin), de métissage culturel (par le poète et ancien Président de la République du Sénégal L. Sédar Senghor : 《Le métissage est un idéal de civilisation 》), de 《 dialogue des civilisations » (notion présente dans toute l'œuvre du philosophe F. Jullien).

${ }^{20}$ Parmi de nombreux exemples : la peinture et la calligraphie (F. Verdier (2007) Entre ciel et terre, Albin Michel, Paris ; (2003) Passagère du silence, Albin Michel) ; la religion (L. Obadia (2007) Le bouddhisme en Occident, La Découverte, Paris).

${ }^{21}$ Parmi de nombreux exemples : la philosophie (A. Cheng, 1997, Histoire de la pensée chinoise, éditions du Seuil; édition révisée et mise à jour en 2002 et (2007) La Pensée en Chine aujourd'hui, Gallimard) ; l'histoire (E.H. Schafer, 1963, The Golden Peaches of Samarkand : A Study of T'ang Exotics, University of California Press, Berkeley. Cf. aussi B. Laufer, anthropologue américain, sur les échanges culturels entre la Chine ancienne et l'Iran) ; l'archéologie (M. Pirazzoli-T’Serstevens, 1994, « Pour une archéologie des échanges. Apports étrangers en Chine - transmission, réception, assimilation », Arts asiatiques, Tome 49, p. 21-33); l'architecture (B. Bosker, 2013, Original Copies : Architectural Mimicry in Contemporary China, University of Hawai'i Press ou les divers travaux de G. Giroir sur les gated communities en Chine).
}

et sages taoïstes de la vieille Chine, symbole de la Longue Marche révolutionnaire dans les années 1930, et boisson indispensable des banquets officiels. En outre, « si une partie des Chinois s'est mise à boire du vin, c'est en quelque sorte malgré son goût » pour ses vertus supposées en termes de santé et de longévité. « Cette boisson étrangère n'est pas adoptée telle quelle, elle subit ainsi un processus de filtrage, de réappropriation, voire d'assimilation culturelle $»$.

\section{Pour une «archéologie » du fait viti-vinicole en Chine : essai d'approche systémique}

Le fait viti-vinicole ou viticolité représente un ensemble d'éléments matériels et immatériels (cf. plus haut). En matière historique, plusieurs méthodes d'approche sont possibles. Les analyses classiques sont de type narratif et s'apparentent à un récit le plus souvent linéaire [7]. On tentera ici de mener une approche différente d'ordre un peu plus théorique, en partie inspirée de l' « archéologie » proposée par M. Foucault, associée à une approche systémique et multiscalaire ${ }^{22}$. Ainsi, on essaiera de faire apparaître à plusieurs échelles les grandes étapes de construction du système viti-vinicole en Chine et leurs discontinuités, en insistant notamment pour les trente dernières années sur les notions de transition, d'émergence et de diffusion.

\section{1. Échelle macro : étapes majeures de construction du système viti-vinicole chinois}

L'histoire de la vigne et du vin en Chine présente une grande ancienneté mais la viti-viniculture chinoise moderne ne remonte qu'à la fin du $19^{\mathrm{e}}$ siècle et, après une longue phase de déclin, la Chine peut aussi être considérée comme un pays émergent en matière viti-vinicole. Les modalités temporelles de cette dynamique du système vitivinicole chinois présentent donc deux traits spécifiques majeurs : la discontinuité et la cyclicité. En effet, l'histoire du vignoble chinois a été faite de phases d'expansion puis de contraction.

Des traces archéologiques de vin de raisin remonteraient à l'Âge du Bronze, mais pour l'histoire officielle c'est l'empereur Taizong des Tang (618-907) qui aurait importé l'art du vin auprès des peuples turcophones des oasis d'Asie centrale dans le cadre de la Route de la $\mathrm{Soie}^{23}$. On retrouve déjà ici la dimension interculturelle et une forme de pré-mondialisation. Mais, à cette époque, la consommation était principalement réservée à l'usage de la cour impériale, et notamment pour ses vertus médicinales. Cette première étape consistait principalement dans l'importation de cépages vinifères, donc de matériel biologique.

Dans le domaine de la viti-viniculture moderne, bien des recherches restent à mener. La fin du $19^{\circ}$ siècle a été marquée par la mise en place progressive des

\footnotetext{
${ }^{22}$ M. Foucault (1969) L’Archéologie du savoir, Gallimard, rééd. 1992.

${ }^{23}$ Terme créé en 1877 par le géographe allemand F. von Richthofen.
} 
fondements du système viti-vinicole chinois. De cette époque date la proto-géographie des vignobles et des entreprises viti-vinicoles d'aujourd'hui. La création en 1892 de l'entreprise Changyu à Yantai par un riche homme d'affaires sino-malais, Chang Bishi, a fixé durablement le cœur du système viti-vinicole chinois [8]. Il a également massivement enrichi le matériel biologique de la Chine en important de nombreux cépages européens ou nordaméricains. Les étrangers ont eux aussi contribué à dessiner la première carte des vignobles chinois.

Mais ce premier système viti-vinicole s'est avéré très incomplet. Il s'est caractérisé par l'absence de plusieurs éléments. L'espace productif est resté très concentré sur la province du Shandong. Il s'est agi surtout d'une consommation de vin élitiste assurée par les expatriés (diplomates, hommes d'affaires, religieux...) liés à la colonisation. À l'exception notable de Changyu, les entreprises viti-vinicoles étaient majoritairement à capitaux étrangers. Le fait viti-vinicole s'avérait étroitement lié au fait colonial ou impérialiste. Il s'agissait d'autant plus d'un isolat culturel que nombre d'entreprises viti-vinicoles ont été créées par des missionnaires pour produire du vin de messe. La notion même de tourisme viticole était totalement absente. La qualité du vin était très médiocre.

Au cours de l'époque maoïste, le système viti-vinicole a connu une atrophie progressive. Les entreprises vitivinicoles ont subi une nationalisation forcée. L'influence géopolitique a été profondément néfaste au vignoble chinois. La Chine a été en grande partie coupée du reste $\mathrm{du}$ monde. Le patrimoine biologique viticole ne s'est pas renouvelé, et a sans doute décliné, à l'exception de l'introduction de certains cépages des pays du bloc communiste, comme le Rkatsiteli, issu d'Europe de l'Est. La consommation de vin s'est coupée du peuple : le vin n'a plus été servi que dans certains banquets d'État pour les hauts dirigeants politiques chinois ou étrangers. L'hégémonie des baijiu, et notamment du Moutai, symbole de la Longue Marche, a été quasiment totale, alors que le vin était considéré comme non-national et bourgeois. La production de vin et le vignoble lui-même sont tombés à un niveau très faible à la fin de l'époque maoïste [1]. À la fin des années 1970, le système viti-vinicole chinois se trouve dans une situation d'attrition généralisée.

\subsection{L'émergence d'un système viti-vinicole à partir des années 1980 et ses limites}

Depuis une trentaine d'années, mais surtout les années 2000, la Chine enregistre une véritable « fièvre du vin » (putaojiu re) [9]. De nombreux éléments du système viti-vinicole se mettent en place souvent à grande échelle et à grande vitesse. Chacun de ces éléments mériterait une étude approfondie. Ainsi, la production et la consommation ont connu des progressions parfois spectaculaires.

Au moment où la consommation mondiale de vin tend à baisser (2007 : 255 millions hl ; $2012: 243$ millions), la Chine fait partie des pays dont la consommation de vin a le plus augmenté. Elle est passée de 10,6 millions
Tableau 3. Évolution de la superficie, production et consommation de vin en Chine (2000-2012).

\begin{tabular}{|c|c|c|}
\hline CHINE & 2000 & 2012 (estimations) \\
\hline Vignobles (milliers ha) (table + cuve) & 300 & $570[10]^{24}$ \\
\hline Production de vin (millions hl) & 10 & 14 \\
\hline Consommation de vin (millions hl) & 10 & 17 \\
\hline
\end{tabular}

Source: Statistical report on world vitiviniculture 2013, OIV.

hl en 2000 à 17,8 millions hl en 2012 (France : 30 millions hl ${ }^{25}$. À moyen terme, elle va devenir le premier marché de consommation du monde. Comme souvent en Chine, cette révolution viticole résulte en partie de décisions gouvernementales. En 1987, à l'occasion d'une réunion tenue à Guiyang, la Commission d'État pour l'Économie et le Commerce, le Ministère de l'Agriculture, le Ministère du Commerce et le Ministère de l'Industrie légère décidèrent de réduire les investissements dans les alcools forts (comme le baijiu) et d'encourager les alcools de fruits. Cette réforme dite de Guiyang visait à la fois à limiter les importations de céréales et à favoriser la santé publique.

Les vignobles à vin représentent une part très minoritaire de la superficie cultivée totale et du paysage agraire, sauf localement (ex. Changli, Hebei). Les principales marques sont : Great Wall, Dragon Seal, Changyu, Dynasty, Imperial Court. La production de vin a connu une augmentation très significative mais moindre, de l'ordre d'un tiers en passant de 10,5 millions d'hl en 2000 à 14,8 millions en 2012 (France : 41 millions hl) plaçant la Chine au 5ème rang mondial, devant l'Australie.

Les acteurs du système viti-vinicole sont encore assez mal connus. Parmi eux, les meilleurs œnologues, le plus souvent européens ${ }^{26}$, ont été recrutés par les domaines viticoles chinois. Des recherches à cette échelle biographique pourraient permettre de connaitre le rôle passé ou actuel parfois déterminant de ces « experts nomades » dans la construction des vignobles chinois et l'émergence d'une culture du vin et d'élaborer des typologies. De même, la reproduction plus ou moins fidèle de l'architecture des châteaux français ou italiens a représenté l'une des formes les plus spectaculaires de transplantation de la culture européenne du vin. Mais la typologie des styles architecturaux des châteaux viti-vinicoles en Chine reste à établir, de même que l'identification des statuts, fonctions et usages de ces châteaux. L'oenotourisme a connu un essor remarquable, avec même parfois l'édification de parcs à thème vitivinicoles sans équivalent dans le reste du monde. Là aussi, il y a de riches matériaux pour réaliser des inventaires et des analyses interprétatives de ces formes parfois hors-normes. Foires au vin et festivals du vin se sont

\footnotetext{
${ }^{24}$ Dont 67329 ha pour les seuls vignobles à vin (2007).

${ }^{25}$ Données issues du Statistical Report on World Vitiviniculture 2013 publié par l'Organisation Internationale du Vin, OIV (en anglais : IOV : International Organisation of Vine and Wine).

${ }^{26}$ Parmi d'autres : Alberto Antonini, Denis Boubals, Gérard Colin, Michel Dubourdieu, Laurenz Moser, Fred Nauleau, Michel Roland.
} 
multipliés, par exemple dans la ville de Yantai, mais aussi à Chengdu, Pékin, Shanghai ou Hong Kong. Des musées du vin ont également vu le jour, comme à Yantai (musée Changyu) mais aussi dans la Beijing Winery, à Macao (1995) ou encore à Qingdao (2010). Les particularités de la muséographie viti-vinicole en Chine restent à identifier.

La mise en relation de la consommation et de la production locale ou importée s'opère à travers un système de distribution multicanaux venu bouleverser la géographie du commerce mondial du vin. Depuis l'abolition des taxes en 2008, Hong Kong est ainsi devenu l'un des hubs mondiaux de commercialisation du vin. Les ventes aux enchères de grands crus organisées par Sotheby's et Christie's y pulvérisent des records. En Chine continentale, le vin est distribué dans des boutiques indépendantes, des grandes surfaces (premier supermarché du vin créé à Shanghai en septembre 2011) ou des foires. Les bars à vin et les clubs de vin se multiplient dans les villes. L'e-commerce est en plein boom, avec plus d'une centaine de online wine sellers. Progressivement, le réseau de distribution ne se cantonne plus dans les mégapoles, notamment Shanghai, mais se diffuse dans des villes de taille plus modeste, comme Wenzhou dont l'activisme de la diaspora a permis un véritable bond des importations de vin.

Ce système viti-vinicole émergent s'appuie sur une base productive de plus en plus étendue. Pour autant, la géographie des vignobles chinois à échelle fine reste floue et très évolutive. Les statistiques restent peu fiables et peu accessibles. Pour répondre à la demande en pleine explosion, la surface viticole chinoise a quasiment doublé en dix ans en passant de 300000 ha en 2000 à 570000 ha en 2012 (vignoble français : 800000 ha). Dès 2002, la superficie viticole (raisin de bouche et raisin de cuve) de la Chine a dépassé celle des États-Unis (150 000 ha supplémentaires). Cet ensemble de vignobles est principalement concentré dans les régions au climat sec de la moitié nord de la Chine : Shandong, Xinjiang, Hebei, Henan, Liaoning, Jilin, Shanxi, Gansu, Ningxia, Mongolie intérieure, mais certains vignobles tropicaux d'altitude (ex. Yunnan) se développent également. À peine au $20^{\text {ème }}$ rang mondial en 1980, le vignoble chinois occupe désormais le $5^{\text {ème }}$ rang, devant la Turquie.

La Chine montre aussi une progression régulière de la part des vins de qualité, avec même l'apparition de vins parfois haut de gamme. Parmi les vignobles ou vins prestigieux, on peut citer Château Junding de Yantai (Shandong), Huadong Parry de Qingdao, Niya Vintage 2008, Guoan de Citic au Xinjiang ainsi que plusieurs crus au Ningxia (Château Changyu Moser XV) et au Shanxi ; dans cette province, Grace Vineyard a été créé en 1997 par un riche Hongkongais avec l'aide du célèbre œnologue languedocien Denis Boubals. L'un de ses grands crus a obtenu le nombre maximal de points de la part du célèbre Robert Palmer's Wine Advocate. La Chine excelle aussi dans le domaine des vins de glace, notamment au Liaoning $^{27}$.

\footnotetext{
${ }^{27}$ Ainsi, Berry Bros. \& Rudd, plus ancien négociant britannique en vins et spiritueux, est devenu le premier détaillant du pays à proposer de manière permanente quatre grands crus chinois (trois vins de glace et un mélange de Cabernet Sauvignon et de
}

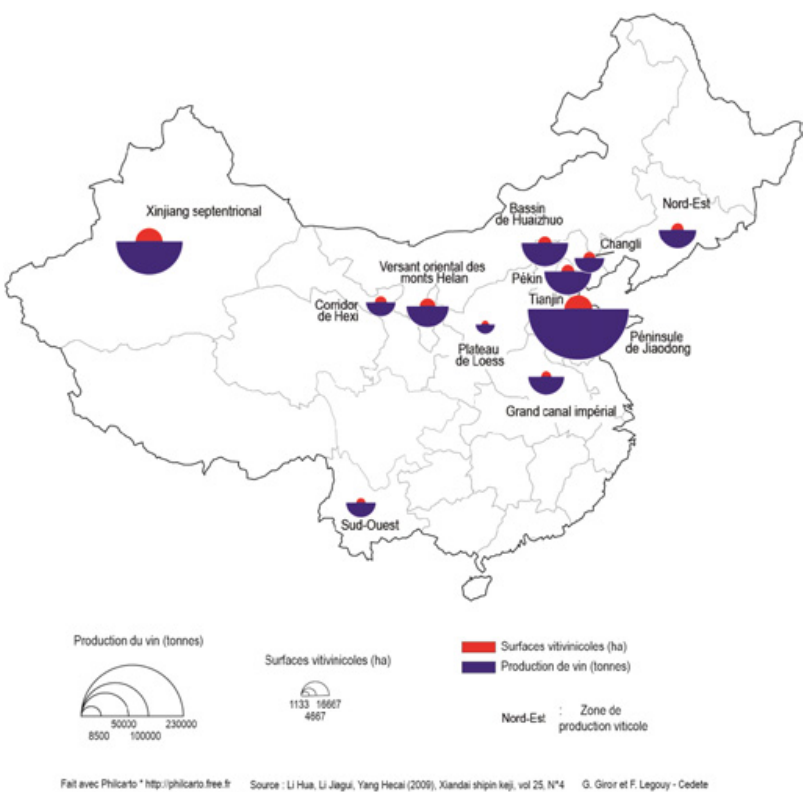

Figure 2. Géographie du vignoble à vin en Chine.

De nouveaux vignobles se multiplient. L'insertion de nouveaux territoires viti-vinicoles dans un espace donné crée inévitablement des interactions pouvant donner lieu à des conflits d'usage du sol. Ainsi, le boom du vin en Chine est à l'origine d'importants enjeux fonciers encore à peine décrits. La demande de vin en Chine, satisfaite majoritairement par les vins locaux, suppose l'extension des surfaces viticoles. Or, la création de nouveaux vignobles en Chine s'opère au détriment des formes d'utilisation du sol existantes, agricoles ou non. Indirectement, la fièvre de consommation du vin débouche ainsi sur des conflits d'usage du sol. Certains nouveaux vignobles entraînent des conflits avec les aires protégées. Ainsi, les projets d'extension ou de création de vignobles sur les bas versants et piémonts des montagnes du Sichuan ou du Shaanxi se traduisent par des défrichements forestiers ; or, certaines d'entre eux sont situés en périphérie des parcs nationaux où vivent les pandas [4]. Ailleurs, la création de nouveaux vignobles obéit à une logique spéculative. Ainsi, sur le littoral de la péninsule du Shandong, la création d'un vignoble peut s'inscrire dans une stratégie de sécurisation de terrains non loin de la mer en vue d'une future revente avec plus-value. La création d'immenses périmètres viti-vinicoles dans le désert de Gobi pose aussi des défis en termes de développement durable.

La construction accélérée de ce système viti-vinicole s'appuie sur de lourds investissements. L'une des expressions territoriales majeures de cette mutation économique, paysagère et culturelle est représentée par la multiplication des châteaux viticoles (jiuzhuang) de type européen,

Merlot, Château Changyu Moser 2008 XV, région autonome Hui du Ningxia) : cf. G. Collins (2013) « Berry Bros. \& Rudd Stocks Chinese Wine From Château Changyu », Bloomberg.com, 20 mars. 
qui associent parfois production et oenotourisme ${ }^{28}$. Ces lourds investissements ont été assurés par des entreprises privées (Changyu), publiques (Cofco/Zhongliang), mixtes (Dynasty/Wangchao) ou étrangères. Il n'est pas rare d'observer des nouveaux riches chinois créer des domaines viti-vinicoles dans leur région : ainsi, certains promoteurs immobiliers de la région de Penglai investissent dans la vigne associée à d'imposants châteaux de type européen. De plus en plus d'investisseurs chinois rachètent des vignobles à l'étranger (France, Afrique du Sud...). Des enquêtes sur ces néo-vignerons feraient apparaître leur profil socio-professionnel, leurs motivations et leurs modèles. Cependant, si certains éléments constitutifs d'un système viti-vinicole se mettent en place, d'autres font défaut. Encore au début des années 2000, G. Giroir pouvait conclure à l' « absence de système territorial vinicole en Chine »[1]. L'augmentation de la production ne s'est pas traduite par l'émergence d'une société de vignerons, ou de villages de vignerons. Il y a un décalage entre la sphère productive et la sphère sociale et culturelle. Il n'y a pas encore en Chine de société viti-vinicole. De même, le tourisme viti-vinicole reste très concentré dans des châteaux viticoles puissants mais isolés, sans effet d'entraînement sur le développement économique du milieu rural local ; en Chine, on est encore loin des régions oenotouristiques et des routes des vins observables en Europe notamment.

La consommation de vin globale s'accroît rapidement, mais la consommation par adulte reste l'une des plus faibles du monde, comparable à celle de certains pays musulmans, même si elle progresse (environ 2 litres prévus en 2015 ; France ou Italie : 50 litres ; États-Unis : 13 litres). L'importation massive des cépages européens ne s'est pas traduite par celle du système de classement des crus. Il n'y a pas de hiérarchie ni de certification des vins comme on peut en trouver en France ou en Italie. La difficile émergence d'un système de certification des vins en Chine représente un sujet de recherche en soi.

De même, paradoxalement, la Chine est devenue en une décennie un grand pays de vin, mais elle ne fait toujours pas partie des 44 États membres de l'OIV; elle n'y apparaît qu'à travers le statut d'observateurs que possèdent la ville de Yantai et la région autonome Hui du Ningxia, autorisées à participer aux travaux de l'OIV.

Alors même que le marché chinois était perçu comme le nouvel Eldorado du vin, les dernières statistiques montrent un ralentissement. Ainsi, la production de vin a baissé de près de $15 \%$ entre 2012 et 2013 pour tomber à 11,7 millions hl, soit un niveau équivalent à celui de l'Afrique du Sud ou du Chili ${ }^{29}$. Cette crise de croissance et les difficultés du leader Changyu montrent la relative fragilité du système viti-vinicole chinois. En effet, depuis les années 2010, les effets du ralentissement économique en Chine mais aussi la campagne anticorruption menée par les autorités ciblée contre les banquets et les achats somptuaires ont pesé sur la demande intérieure. Ils rappellent la prégnance du facteur politique.

\footnotetext{
${ }^{28}$ Cf. l'inauguration en 2016 du plus important parc à thème mondial sur le vin (Yantai).

29 Données OIV : «Éléments de conjoncture viticole : le secteur du vin retrouve son équilibre », avril 2014
}

Tableau 4. Baisse des performances financières de l'entreprise Changyu depuis 2011.

\begin{tabular}{|l|c|c|}
\hline Date & $\begin{array}{c}\text { Chiffre d'affaires } \\
\text { (milliards yuan) }\end{array}$ & $\begin{array}{c}\text { Bénéfice net } \\
\text { (milliard yuan) }\end{array}$ \\
\hline 31 décembre 2010 & 4,9 & 1,4 \\
\hline 31 décembre 2011 & 6 & 1,9 \\
\hline 31 décembre 2012 & 5,6 & 1,7 \\
\hline 31 décembre 2013 & 4,3 & 1 \\
\hline
\end{tabular}

Source : Bloomberg Businessweek, 30 avril 2014.

La découverte de traces de fongicides dans des vins Changyu et la prolifération incontrôlée des contrefaçons de grands crus étrangers fragilisent la confiance du consommateur. La concurrence des vins étrangers s'est considérablement renforcée : la part des importations est ainsi passée d'à peine $10 \%$ début 2000 à un quart du marché fin 2012. La concurrence provient également des châteaux viticoles implantés par des Européens en Chine : ainsi, en avril 2013, JV Domaines Baron de Rothschild/CITIC a créé un château viticole de 50 ha et d'une capacité de 20000 bouteilles par an à Penglai, en plein cœur du fief de Changyu. Le résultat de toutes ces tendances négatives est une baisse sévère des performances financières de l'entreprise Changyu depuis 2011 ; ainsi, malgré l'augmentation de sa production, le chiffre d'affaires à la fin de 2013 est sensiblement inférieur à celui de la fin 2010, effaçant ainsi trois ans de croissance du marché.

$\mathrm{Au}$ total, dans le domaine du vin comme dans d'autres, le marché chinois apparaît en pleine transition et émergence. Le vin lui aussi est pris entre euphorie et turbulences du marché.

\section{3. À échelle micro : monographies d'entreprises viti-vinicoles et de cépages}

Encore rares sont les recherches portant sur la trajectoire des entreprises viti-vinicoles en Chine, ou bien elles font l'objet d'un récit succinct ou fragmentaire. Or, l'étude de l'origine et de l'évolution de ces entreprises permettrait d'ouvrir un vaste champ de recherche. À travers ces monographies d'entreprises, il serait possible d'appréhender de l'intérieur le monde du vin en Chine.

\subsubsection{Trajectoires des entreprises viti-vinicoles}

L'analyse détaillée de l'entreprise pionnière Changyu (Yantai) par G. Giroir [8] a permis de combler en partie ce manque. Depuis le début des années 2000, la production, la consommation de vin mais aussi l'oenotourisme ont connu une très forte expansion en Chine. Ce phénomène est observé ici à travers le cas de l'entreprise Yantai Changyu Pioneer Wine Co. Ltd., (en abréviation ici Changyu), leader historique en Chine créé en 1892 et devenu l'une des principales entreprises viti-vinicoles mondiales. Changyu constitue aussi l'inventeur des « châteaux viticoles » (jiuzhuang) en Chine et y exerce un rôle structurant dans l'essor de la production viti-vinicole et de l'oenotourisme. L'objectif de cette étude a été de montrer, à plusieurs échelles, le rôle majeur de Changyu dans la construction du système viticole chinois et son intégration rapide dans 
la mondialisation. Cette étude s'est appuyée sur une série d'enquêtes de terrain menées en octobre et décembre 2012 (Yantai, Pékin). L'étude retrace d'abord les étapes historiques majeures de développement de Changyu. La deuxième partie, à l'échelle urbaine et intra-urbaine, analyse les formes d'inscription territoriale de l'entreprise Changyu à Yantai et Penglai (Shandong). Enfin, à une échelle micro-territoriale, ont été présentés l'archipel des châteaux Changyu dans diverses provinces chinoises (Pékin, Liaoning, Shaanxi, Ningxia, Xinjiang) puis son réseau de châteaux viticoles à l'étranger. La conclusion s'interroge sur la validité et les limites du modèle de développement de Changyu, en crise depuis 2011.

D'autres cas d'entreprises peuvent être analysés. Ainsi, l'actuelle Beijing Winery (Beijing xijiao putaojiu chang) a été créée par un Frère Mariste, prêtre catholique français, en 1910 pour produire du vin de messe. Le musée de l'entreprise actuelle comporte la statue du prêtre fondateur. Le cimetière de l'église Heishanhu a été converti en vignoble. La cave a été creusée sous l'église. L'entreprise a été implantée à Fuwai, près des monts Yuquan, dans la banlieue ouest de Pékin, à proximité du Palais d'été. La production comprise entre 5 et $6 \mathrm{t}$ était assurée par des catholiques locaux. En 1946, l'église enregistra officiellement l'entreprise viti-vinicole sous le nom de «La Shangyi Cave de Pékin (Shangyi Winery of Beijing ») et commença à vendre du vin sur le marché chinois, notamment auprès des ambassades étrangères, des hôtels et des compagnies de navigation ; une partie était exportée en Europe. Après cette première période religieuse, une deuxième phase s'ouvre avec la nationalisation de l'entreprise par l'État en 1949 ; l'entreprise produisait moins de $10 \mathrm{hl}$ avec 13 employés. En 1953, le nom a été changé pour Beijing Shangyi Winery et l'église catholique cessa de s'impliquer dans la production. En 1959, elle a été rebaptisée Beijing Winery par le gouvernement chinois et déménagea dans la rue Yuquan. Une troisième période correspond à la globalisation de l'entreprise. Ainsi, en 1987, une filiale a été créée (Beijing Dragon Seal Winery) pour introduire les techniques œnologiques françaises. Un domaine viticole a été implanté à partir d'une dizaine de cépages français (notamment Cabernet Sauvignon et Chardonnay) dans le district de Huailai (province du Hebei) à $150 \mathrm{~km}$ au nord-ouest de Pékin. La première bouteille a été commercialisée en 1988. En 2006, Dragon Seal devint le premier vin chinois à l'export et représenta près de $40 \%$ du vin chinois exporté.

L'entreprise viti-vinicole Tonghua a été créée par les Japonais en 1937 dans la province du Jilin. Longtemps servi à l'occasion des banquets d'État, le vin rouge Tonghua est issu des vignes sauvages des monts Changbai. Plus récemment, certains vignobles ont été créés par des investisseurs étrangers (ex. Huadong Perry à Qingdao ; Reifeng-Auzias ou Domaines Baron de Rotschild à Penglai ; Bodega-Langes par le propriétaire de l'entreprise Swarovski à Changli....).

\subsubsection{Trajectoires des cépages}

L'origine et les modalités de diffusion des cépages étrangers en Chine constituent un champ de recherche à part entière. De fait, la Chine est devenue une terre de concentration exceptionnelle de cépages importés. Depuis la fin du $19^{\mathrm{e}}$ siècle, elle a connu une puissante vague d'importation de cépages principalement européens mais aussi d'autres continents. Selon Hong Guangzhu (2001) [11], au cours de la période 1892-1962, 77 cépages différents ont été introduits en Chine depuis la France, l'Italie, l'Allemagne, l'Autriche, la Suisse, la Bulgarie, la Roumanie, la Hongrie, la Russie, les États-Unis, la Grande-Bretagne, la Turquie, l'Iran et le Japon.

Mais l'histoire, et peut-être encore davantage la géographie de ces cépages allogènes restent à faire. Car, au-delà des aspects strictement ampélographiques, chaque cépage a une trajectoire et une identité particulières qui le fait relever également du champ des sciences humaines et sociales.

Certains cépages ont des trajectoires singulières. C'est le cas par exemple du cépage français Miel de rose qui a été implanté au Yunnan par des missionnaires français au milieu du $19^{\mathrm{e}}$ siècle ; suite à sa disparition en France du fait du phylloxéra, il ne subsiste plus que dans une petite communauté catholique au sud-est de Kunming.

D'autres cépages ont suscité de multiples débats. C'est notamment le cas du Cabernet Gernischt. Importé également de France à la fin du $19^{\mathrm{e}}$ siècle, il a longtemps été considéré soit comme un Cabernet Franc, soit comme un mélange de Cabernet Franc et de Cabernet Sauvignon. En 2012, une analyse ADN réalisée par le célèbre généticien des vignes originaire du Valais suisse, José Vouillamoz, a montré qu'il s'agissait en réalité du Carmenère, vieux cépage bordelais quasiment disparu de France mais emblématique du vignoble chilien, et représenté en Chine principalement dans les provinces du Shandong et du Ningxia ${ }^{30}$. Il pourrait y devenir l'un des marqueurs des vins rouges de qualité.

\section{3. Études empiriques à échelle micro-territoriale des « châteaux viti-vinicoles $»$}

À une échelle micro-territoriale, les études de cas élaborées à partir d'enquêtes de terrain apportent une masse considérable de données qualitatives, bien que souvent fragmentaires et incertaines. À travers les entretiens, elles donnent la parole aux acteurs du système viti-vinicole. Elles permettent de mener des approches pluridisciplinaires associant géographie, histoire, architecture, paysagisme, aménagement, tourisme, sociologie, marketing, sémiologie...

L'apparition de paysages productifs et architecturaux de type principalement européen exprime et se traduit par la montée en puissance de l'oenotourisme, comme le montre l'inauguration en 2016 du plus important parc à thème mondial sur le vin à Yantai.

\footnotetext{
$\overline{30}$ J. Robinson, J. Harding, J. Vouillamoz (2012) Wine Grapes : A complete guide to 1,368 vine varieties, including their origins and flavours, Peguin : Londres. Cf. aussi Hong X. M., Yao Y. X., Du Y. P., Zhai H. (2012) «"Cabernet Gernischt" is most likely to be "Carmenère" », Vitis, 51 (3), p. 125-127.
} 
Les relations entre médias et vin représentent à elles seules un champ d'investigation à la fois nouveau et très fécond. De plus en plus de séries télévisées (Wine Beauty, 2014 : Hongjiu qiaojia ren) ou de films (Eternal Moment, 2011 : Jiang aiqing jinxing dao daodi) prennent le monde du vin pour thème ou décor. Ils associent souvent des acteurs de Taiwan, de Hong Kong et de Chine continentale; ces drames familiaux ou romantiques se déroulent en Chine ou dans des hautslieux du vin comme Bordeaux. Les relations entre vin et art (étiquettes, calligraphie, expositions, objets...) sont également très denses en Chine ; leur exploration ne fait que commencer ${ }^{31}$.

\section{1. Études de châteaux viti-vinicoles à Yantai (province du Shandong)}

Trois études de cas réalisées par G. Giroir permettent de montrer les types d'approche possibles.

\subsubsection{Chateau Changyu-Castel}

Le château Changyu-Castel représente le modèle historique et de référence des châteaux viticoles en Chine. Il invite à développer une analyse de type microterritoriale mettant au jour la différenciation ainsi que le fonctionnement internes de ce type d'espace à la fois productif et récréatif. Ce château viti-vinicole a servi de modèle à la plupart des autres châteaux viti-vinicoles construits en Chine ces dernières années ; il permet aussi d'élaborer des approches comparatives et typologiques des châteaux viti-vinicoles chinois.

Créé conjointement en 2002 par l'entreprise Changyu et le groupe français Castel et situé dans le village Beiyujia dans la Zone de Développement Économique et Technique de Yantai (Yantai kaifaqu), il se compose d'un vaste château entouré par 135 ha de vignobles, notamment issus du cépage Cabernet Gernischt. Le château abrite les ateliers de production du vin, les caves à vin, le centre de recherches et de développement des plants de raisin, le centre des échanges culturels autour du vin, la boutique et des restaurants.

À l'origine, il s'agit d'un partenariat entre l'entreprise locale Changyu et le groupe français Castel. Le château Changyu-Castel a été conçu et décoré par Marcel Mirande ${ }^{32}$, architecte spécialisé dans les bâtiments viticoles (chais, châteaux) et lié au groupe Castel. En échange, Changyu devait distribuer les vins Castel à travers son réseau. Mais, deux ans plus tard, Changyu n'avait pas vendu une seule bouteille! Toutefois, en s'associant

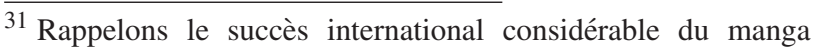
japonais inspiré par le monde du vin Drops of God. Cf. aussi plus bas le rôle de l'artiste plasticien Zeng Fanzhi.

${ }^{32}$ M. Mirande (2004) « Architecture et conception des bâtiments viticoles 》, Revue des Enologues, n 113 et 2009 « Architecture et Éco-conception des bâtiments viticoles », $\mathrm{n}^{\circ} 133$. Surtout 1 'ouvrage Architecture des bâtiments viticoles. De la conception à la réalisation (2012).
}



Figure 3. Plan du château Changyu-Castel (Yantai). Cliché G. Giroir, octobre 2012.

avec d'autres distributeurs, le groupe Castel est devenu le premier importateur de vins en Chine. Castel reste actionnaire de $30 \%$ de Changyu \& Castel Estate. La stratégie de Changyu-Castel repose sur la vente d'un vin de milieu de gamme et un oenotourisme de masse.

Globalement, la logique de Château Changyu-Castel est celle d'un parc à thème dédié au vin. Il s'agit d'un territoire fermé par une enceinte et sécurisé par des gardes en uniforme. Il faut acheter un ticket pour pouvoir entrer. Le plan du château viticole exprimé en trois langues (chinois, anglais, coréen) montre un microterritoire organisé selon trois principes : un élément de centralité (le château proprement dit), un dualisme sectoriel (parc viticole devant et derrière le château) et divers éléments de détail linéaires ou ponctuels (couloirs viticoles, places, plateforme...). Le touriste est censé parcourir ce micro-système territorial viticole à travers des circuits viticoles (Changyu jiuzhuang lüyou xianlu) soit en plein air soit à l'intérieur même du château.

Le « parc viticole» (putao gongyuan) devant le château comprend plusieurs éléments, notamment un « corridor viticole de style français » (tonnelle), un « corridor de dégustation de raisin » et des «fenêtres sur la ville du vin $\gg$ (ensemble de $2000 \mathrm{~m}^{2}$ englobant un centre d'exposition du vin, des salles d'expérience personnelle du vin et un restaurant à thème autour du vin). Quant à lui, le parc viticole à l'arrière représente principalement une aire de loisirs (pique-nique, promenade, repos au bord de la rivière Canglang et du lac Liquan ... ) ; elle est agrémentée par la plate-forme Yingbin, endroit surélevé qui permet de voir le château et le vignoble dans son ensemble, ainsi que par deux places (Haina et Putao shaonu) contribuant à renforcer l'ambiance romantique.

Le fonctionnement de ce système oenotouristique repose sur la volonté de canaliser les flux de touristes, sur un souci didactique (ex. rangées de cépages étrangers en version bilingue, le plus souvent français et chinois) et une stratégie de maximisation des profits à travers la vente de vin. Cette logique de parc à thème se trouvera fortement amplifiée par l'achèvement de la construction de la future « ville internationale du vin ». 


\subsubsection{Junding}

Le château viticole Junding a été construit en mars 2005 et ouvert au public en septembre $2007^{33}$. Il appartient au puissant groupe COFCO, qui y a investi pas moins de 300 millions de yuan $(47,1 \text { millions de } \$)^{34}$. Il occupe une superficie totale d'environ $6 \mathrm{~km}^{2}$. La production de vin et l'oenotourisme s'inscrivent résolument dans une stratégie haut de gamme, voire de luxe. Avant la création de ce vignoble s'étendaient autrefois des champs de maïs, de cacahuètes et de blé. Près du château subsistent curieusement quelques parcelles de maïs. Les paysans locaux ont été expulsés. La centaine de familles ont été relogées dans de petits immeubles de trois villages (cun). Le vignoble en propre s'étend sur 400 ha $(6000 \mathrm{mu})$ dans la Nava Valley. À cela s'ajoutent également des parcelles de vigne sous contrat en dehors du domaine. Le rendement moyen est de $400 \mathrm{~kg}$ par $m u$. Dans les vignobles sous contrat, la production est soumise à un strict contrôle de qualité, notamment sur le taux de sucre. En cas de mauvaise année, Junding compense le manque à gagner des paysans, ce qui permet de maintenir un niveau constant de qualité. Au total, Junding est élaboré exclusivement avec des raisins locaux. Il peut être considéré comme un vin de terroir.

Le vignoble a été créé à partir de cépages importés de France, d'Italie et d'Allemagne. Le Cabernet-Sauvignon représente le principal cépage pour le vin rouge. Les vins blancs sont issus de cépages Riesling et Chardonnay. Un centre de recherche local s'efforce d'adapter les cépages importés à l'environnement local. Château Junding bénéfice de conditions exceptionnellement favorables à la viticulture de qualité. Comme le vignoble de Penglai dans son ensemble, le vignoble de Junding bénéficie des « trois $\mathrm{S} \gg$ (sun, sea and sand) (soleil, mer et sable). Mais s'y ajoutent d'autres facteurs spécifiques favorables. Junding bénéficie d'un micro-climat. Le centre du vignoble est occupé par le lac Phoenix (Fenghuang), vaste réservoir artificiel qui alimente en eau potable la ville de Penglai. Cette étendue d'eau douce constitue une réserve de chaleur favorable à la croissance de la vigne. Sa surface entraîne un phénomène de réverbération contribuant lui aussi à augmenter luminosité et températures. Les sols sont en partie sableux, mais de surcroît en creusant le sol, des blocs de jade auraient été trouvés ${ }^{35}$. Le contact entre les racines de la vigne et le jade confèrerait au vin local une saveur toute particulière. Au-delà de cet aspect gustatif présumé, le jade comporte une forte dimension symbolique. En Chine, le jade incarne le pouvoir. C'est aussi un symbole de vie éternelle et de résurrection.

La marque Junding est traitée comme une marque de luxe. Le nom Junding lui-même symbolise cette stratégie : il signifie « chapeau de l'empereur » (jun : empereur ;

\footnotetext{
${ }^{33}$ La plupart des données proviennent d'un entretien mené en décembre 2012 auprès du gestionnaire de Château Junding, M. Xu Shimin.

${ }^{34}$ Wang Qian (2012) « Grapes of class : Rising chateau county in Yantai », China Daily, 28 juin.

${ }^{35}$ A noter l'existence d'une Jade Valley Wine \& Resort créée en 2000 sur le versant nord des monts Qinling, près de Xi' an, district de Lantian (province du Shaanxi).
}

ding : chapeau). Le poème « Junling tianxian, wending dongfang » signifie qu'il ne s'agit pas seulement du chapeau de l'empereur, mais que ce chapeau a vocation à devenir le chapeau du monde entier. Les prix ont été fixés à un niveau très élevé. La bouteille la moins chère (Dongfang) ne coûte pas moins de 1819 yuan (2012). Certaines bouteilles mises en réserve lors de l'inauguration (yuan nian) sont les plus chères de la production. Deux bouteilles offertes en cadeau à Henry Kissinger valent 31999 yuan. Dans le cadre de son positionnement vers le luxe, Junding préfère limiter la production de vin. Le luxe s'exprime jusque dans les étiquettes. L'association avec un célèbre peintre contemporain, Zeng Fanzhi ${ }^{36}$, a permis d'élaborer des étiquettes artistiques pour les bouteilles de réserve.

La logique de club a été poussée assez loin. Junding a créé un club dans la plupart des grandes villes chinoises. Le club comprend des célébrités globales telles que Céline Dion ou le Prince Charles. Des spectacles, notamment de musique classique, sont proposés pour les seuls membres du club. La fréquence de ces soirées est cependant limitée à environ deux soirées par an. La raison avancée est que le parfum des femmes serait nocif pour le vin. Junding possède une cave creusée à $11 \mathrm{~m}$ sous terre $(70 \%$ d'humidité, $15^{\circ} \mathrm{C}$ de température moyenne). Les tonneaux viennent de France. Une partie est réservée à des caves privées ; pour bénéficier du privilège d'y entreposer son vin, il faut d'abord être membre du club, puis acheter l'équivalent d'un tonneau, donc de 300 bouteilles. Le souci didactique est très présent. La salle de dégustation comporte un panneau présentant les arômes et les couleurs pour éduquer les visiteurs au monde du vin.

Le paysage viticole et architectural de Junding est de grande qualité. Le vignoble bénéficie d'un traitement paysager très soigné, notamment sous la forme d'arbres fruitiers ou décoratifs. Gingkos, lilas, acacias, acacias pourpres, mûriers, cerisiers et kakis parsemés dans le vignoble ou autour de l'hôtel permettent de varier le paysage et d'agrémenter les promenades. L'architecture de Château Junding est assez curieusement de type hacienda ${ }^{37}$. Les patrons ont visité de nombreux châteaux en France et en Italie mais ont opté finalement pour le style espagnol, beaucoup plus rare en Chine et pour se différencier des châteaux à la française du groupe Changyu, concurrent direct de COFCO sur le marché du vin.

La stratégie haut de gamme de Château Junding concerne également des formes de tourisme et de loisirs viticoles. En effet, il s'agit du premier château viticole en Chine, voire en Asie à offrir un parcours de golf. Conçu par Bernhard Langer, le golf 18 trous (Château Junding Golf Club ou COFCO Junding Winery Golf Club : Junding Jiuzhuang Gaoerfu Julebu) s'inscrit

\footnotetext{
36 Zeng Fanzhi (1964) : peintre expressionniste, rendu célèbre par la série de toiles « Masques », dont certaines ont battu des records de ventes (9,7 millions de dollars) aux enchères à Hong Kong.

37 L'hacienda correspond à l'origine à une exploitation agricole souvent centrée sur l'olivier et la vigne et occupée par des grands propriétaires. Initailement d'origine andalouse, elle est devenue emblématique de l'Amérique latine.
} 




Figure 4. Junding, château viticole de style hacienda. Cliché G. Giroir, octobre 2012.

dans un environnement de collines s'étendant sur 100 ha (1 $500 \mathrm{mu}$ ), à proximité du lac Phoenix. Un hôtel 5 étoiles d'une capacité limitée à 92 chambres permet le séjour d'une clientèle fortunée. Junding comporte aussi un clubhouse 5 étoiles. Ce château viticole de luxe s'efforce de contenir la fréquentation touristique. Le nombre de touristes atteint toutefois environ 300000 personnes. La plupart viennent de Pékin en voiture ou résident dans la ville de Yantai. Golf et hôtel ont pour objectif principal de faire vendre le vin. L'oenotourisme représente moins de $10 \%$ des bénéfices du domaine. Le vin assure donc l'essentiel des revenus du domaine. Néanmoins, le binôme vignoble-golf sert aussi d'argument marketing fort pour le lancement d'une opération immobilière de luxe. Ainsi, du côté Est, un projet de construction de villas et de châteaux est en cours. En revanche, les abords du lac sont non-constructibles du fait de la loi sur la protection de l'eau.

\subsubsection{Guobin, un château viticole de style chinois}

Guobin (en chinois : Guobin jiuzhuang, ou château viticole Guobin ; en anglais : Château State Guest) est un château viticole dont la construction a été achevée en 2011 et dont l'ouverture au public date de la fin octobre $2012^{38}$. Propriété d'un riche promoteur immobilier

\footnotetext{
${ }^{38}$ La plupart des données proviennent d'entretiens réalisés sur place et de la consultation du site :http://en.stateguest. com/shangu/\&FrontComContent_list01-13091452
}

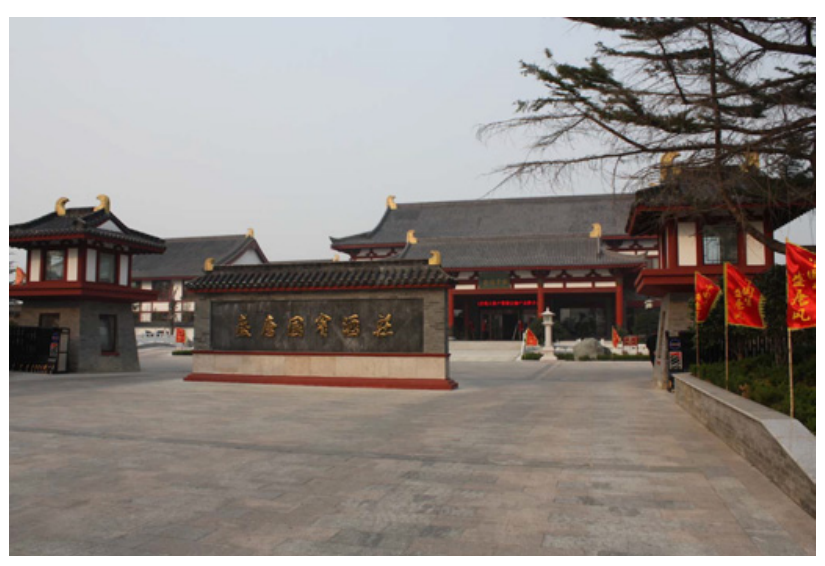

Figure 5. Entrée de Guobin, château viticole de style Tang. Cliché G. Giroir, octobre 2012.

de Penglai, Liu Changting, Guobin représente un cas à part car il s'agit du seul château viticole de style architectural non pas occidental, mais chinois. Parmi les divers styles chinois possible, a été retenu celui de la dynastie Tang. Guobin possède donc une forte identité culturelle et constitue un cas unique au monde. Il s'agit d'un cas remarquable montrant l'importance des relations entre architecture, politique, identité culturelle et nationale dans le fait viti-vinicole en Chine. Il apporte un élément de contradiction aux théories interprétant la diffusion du fait viti-vinicole en Chine comme l'expression de la globalisation, voire de la colonisation de la Chine par une plante de civilisation allogène. Guobin montre au contraire que le fait viti-vinicole peut se trouver réinterprété comme un élément de la culture nationale et même instrumentalisé comme un vecteur de la grandeur de la Chine. En Chine, les relations entre vin et politique, voire géopolitique représentent à elles seules un vaste champ de recherche.

Le choix de la dynastie Tang ne doit rien au hasard. On a choisi le site où l'empereur Taizong (Li Shimin) de la dynastie Tang est venu avec ses troupes à Dengzhou (actuel Penglai). En reconnaissance de leur soutien face aux Coréens, Li Shimin a offert à ses habitants l'art de la vinification. En réminiscence de cet acte historique majeur, les autorités locales actuelles ont planifié le développement d'une Vallée du Grand Empereur Tang ${ }^{39}$, ou Grand Tang Emperor Valley $\left(60 \mathrm{~km}^{2}\right)$, véritable cluster dédié au vin (vignoble de 3333 ha, production de vin de $24500 \mathrm{t}$, accueil de 2 millions de touristes, commerce, loisirs, entreprises viti-vinicoles).

La période des Tang a sans doute été l'un des âges d'or pour la civilisation chinoise. Il s'agit de montrer que le vin n'est pas seulement un produit d'importation occidental, mais est à la fois ancien et chinois. Le message ici est très clair : vin et oenotourisme s'inscrivent dans une logique

\footnotetext{
${ }^{39}$ Ses limites vont de l'ouest du village de Zhaili à $300 \mathrm{~m}$ à l'est du village Guanli, et de la route Zhanbei au sud à la route nationale 206 au nord (incluant le lac Qinglong, la rivière Pingshan, les monts Zhi, Ma et Yu).
} 
d'indigénisation du vin et revêt un caractère politique, voire nationaliste.

La partie construite (3,3 ha) se divise en deux parties : le complexe hôtelier 5 étoiles comportant 63 chambres et un restaurant, et les bâtiments productifs et commerciaux. Dans l'hôtel de luxe centré sur le thème du vin, le marketing est allé jusqu'à systématiser les références à la dynastie Tang. Tout rappelle les Tang. À l'entrée de Guobin, un oriflamme précise « le rêve de la Chine, le style des Tang » (Zhongguo meng, shengtang feng). Sur le sol, parmi les pavés, se trouvent des monnaies des Tang. Dans le hall d'entrée de l'hôtel, un grand panneau en bronze sculpté par un artiste local ayant fait des recherches dans les archives officielles représente diverses scènes expliquant la relation entre la dynastie Tang et le vin local. Diverses peintures et calligraphies évoquent le thème du vin.

Le complexe hôtelier est agrémenté par une maison de thé (yuexin fang) et un bassin de 4000 à 5000 poissons comme dans les familles royales ou l'empereur ; vivant entre 60 et 70 ans, les poissons représentent un symbole de longévité. L'architecture Tang se caractérise par son élégance et sa simplicité. Elle ne comporte pas de peintures ni de petits personnages comme sous les Ming ou les Qing. Les entourages de poignée des portes sont ornés de pivoines stylisées, symbole de la dynastie Tang. Le jardin représente un mélange entre le style de Chine du nord et celui de Chine du sud. Au centre du complexe architectural se trouve un bâtiment à étages (guobin lou) représentant une suite présidentielle. Le prix de la nuit (18 888 yuan) en fait la plus chère de l'hôtel. Des fêtes en costume traditionnel Tang sont périodiquement organisées et se déroulent sur un petit ponton rouge. Des stars de la chanson y participent, notamment originaires de la ville de Xi'an, haut-lieu de la dynastie Tang.

À l'arrière du complexe hôtelier, séparé par une ruelle, se trouve le bâtiment technique, notamment la chaîne d'assemblage avec ses hautes cuves en acier, ainsi que la cave où sont stockées plus de 100000 bouteilles. La production est alimentée par un vignoble de $2000 \mathrm{mu}$ (133,3 ha). Le raisin est acheté en dehors du domaine mais transformé sur place. Dans l'usine, on mélange deux ou trois types de vin selon les avis des techniciens.

Dans la cave, on entend une chanson dont les paroles ont été écrites par le patron et interprétées par un grand chanteur local. Une partie est réservée à des caves privées, seulement numérotées pour préserver l'anonymat de leurs riches propriétaires.

Au milieu d'une petite place intérieure se trouve un faux arbre de grande taille ; sa fonction est de montrer que le vin existe depuis longtemps et que le vignoble local a une longue histoire. Le nom des bouteilles (Sheng Tang) s'inspire directement des Tang. La couleur des étiquettes rappelle les couleurs de la dynastie Tang. On trouve ainsi des bouteilles avec des rubans rouges, jaunes ou verts, conformément aux célèbres « trois couleurs des Tang »(Tang sancai), qu'on trouve notamment sur les céramiques (pièces de vaisselle et statuettes funéraires). De nombreux poèmes Tang ont été placés sur les bouteilles de vin,

\subsection{Approche géo-sémiotique d'un parc à thème viti-vinicole : le cas de Château Changyu AFIP Global (Pékin)}

Dans le contexte du marché émergent du vin, la Chine connaît une forte croissance de l'oenotourisme. Ce phénomène est encore très peu étudié dans la littérature scientifique (Giroir, 2002, 2014). Le cas particulier des parcs à thème sur le vin en Chine n'a pas été décrit à ce jour. Les parcs à thème viti-vinicoles tendent à se multiplier à travers le monde. On peut citer les cas de Lakeridge (Floride) ou de Francis Ford Coppola Winery (Californie) aux États-Unis. L'entreprise viticole Mondavi a également créé the Golden Vine Winery dans le parc Disney California Adventure. La France compte quelques exemples, parmi lesquels le centre oenotouristique Viavino (Saint-Christol, Hérault, près de Montpellier), ou la future Cité des civilisations du vin à Bordeaux prévue pour 2015.

Le principal objectif de cette étude exposée en avril 2014 au congrès annuel de l'Association of American Geographers (Wine Speciality Group, Tampa) a été de présenter les résultats d'une enquête de terrain menée en octobre 2012 dans un parc à thème sur le vin dans la grande banlieue de Pékin, Beijing Château Changyu AFIP Global (Beijing Zhangyu Aifei guoyuan jiuzhuang) ${ }^{40}$.

Au sein de la galaxie des châteaux viticoles Changyu, Beijing Château Changyu AFIP Global représente un cas à part. Il ne peut être considéré comme pionnier dans le domaine de l'oenotourisme en Chine car le château Changyu-Castel à Yantai (province du Shandong) a été créé dès 2002 et représente le modèle oenotouristique historique au sein du groupe Changyu.

Mais en tant que parc à thème viti-vinicole, Beijing Château Changyu AFIP Global représente une forme exceptionnelle d'oenotourisme à un double titre : d'abord, par la rareté des parcs à thème sur le vin dans le monde ; puis et surtout parce que cet oenotourisme s'inscrit dans le contexte culturel de la Chine où le vin ne fait pas partie des modes de consommation traditionnels. Cette altérité conduit à modifier la signification et les fonctions de ce type de micro-territoire. Le cheminement du client chinois s'apparente ainsi à un parcours initiatique d'une civilisation à une autre.

L'approche retenue ici n'est donc pas seulement descriptive ou systémique ; elle représente un essai d'application de la sémiotique narrative à un microterritoire. On définira ici la sémiotique comme l'étude des systèmes structurés de signes signifiants (A.J. Greimas, 1966). Cette méthode d'approche est apparue la plus à même de rendre compte du fonctionnement de ce parc à thème. L'hypothèse principale repose sur l'idée que le parc à thème vitivinicole Beijing Changyu AFIP Global fonctionne comme un espace où se déroule un parcours initiatique visant à convertir le consommateur chinois à un mode de consommation culturellement allogène. Il s'agit de montrer comment le consommateur chinois, buveur traditionnel d'alcools blancs (baijiu), boisson riche en réminiscences taoïstes mais aussi révolutionnaires, est

\footnotetext{
${ }^{40}$ La plupart des informations présentes dans cette étude ont été collectées lors d'une enquête de terrain réalisée sur place en octobre 2012.
} 


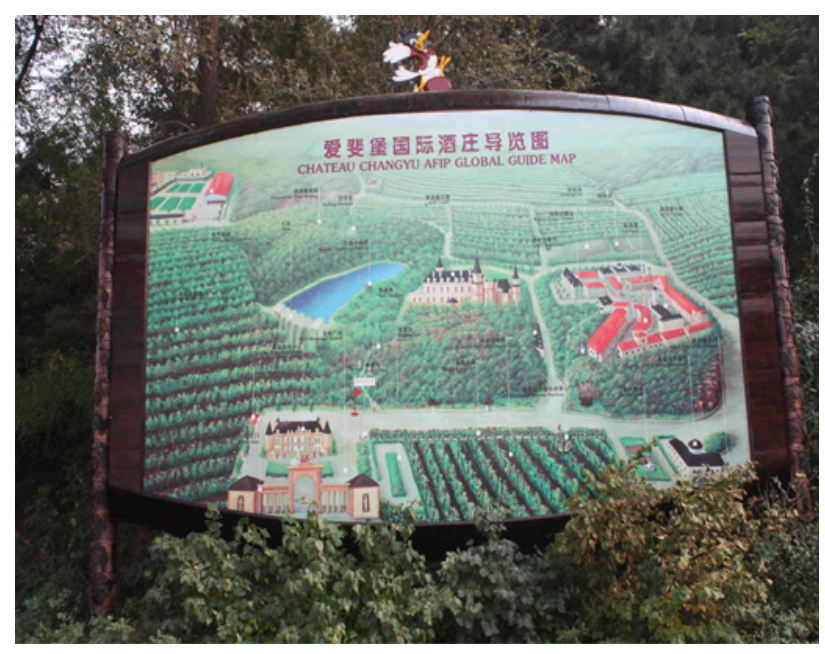

Figure 6. Plan général de Château Changyu AFIP Global. Cliché G. Giroir, octobre 2012.

amené à passer différentes étapes successives destinées à le convertir au vin, boisson jusqu'alors inconnue, d'une autre civilisation, en l'occurrence judéo-chrétienne. Comme dans les rites de passage du profane au sacré (M. Éliade, 1959) ou les parcours initiatiques des héros de contes (A. Van Gennep, 1909 ; V. Propp, 1928), ou plus généralement de tout récit (P. Larivaille, 1974), il y a un état initial, un processus de transformation et un état final. L'idée principale est que le consommateur chinois est une sorte de héros traversant différentes étapes visant à opérer une « conversion de civilisation ». Le parc à thème est conçu comme un opérateur de ce processus. De manière pragmatique, non systématique et non exclusive, la présente approche se revendique donc d'une inspiration géo-sémiotique.

Pour explorer ce parc à thème, ont été d'abord présentés son organisation micro-territoriale et son fonctionnement général dans un essai d'approche géosémiotique centrée sur la notion de parcours initiatique. Une deuxième partie a été consacrée à l'analyse du village européen et à sa fonction majeure dans ce parcours. Dans une troisième partie, les différentes fonctions du château ont été présentées. La dernière partie s'interrogera sur les limites de ce modèle de parc à thème.

Le château Beijing Changyu AFIP Global présente plusieurs spécificités. La situation péri-métropolitaine de Château Changyu AFIP Global, situé à 88 km de Pékin, rend compte de certaines spécificités majeures du parc à thème. Il s'agit du seul des châteaux Changyu à combiner un oenotourisme de masse et un vignoble de grande superficie. À l'inverse de celui de Changyu-Castel, en situation intra-urbaine, celui de Pékin, situé dans le district rural de Miyun, dispose de vastes disponibilités foncières.

Beijing Château Changyu AFIP Global représente l'une des manifestations du processus multiforme d'internationalisation de l'entreprise Changyu engagé depuis le milieu des années 2000. Autrefois détenue par la municipalité de Yantai, l'entreprise a ouvert son capital en 2005 à des entreprises occidentales, notamment italiennes, américaines et portugaises.

Le château Beijing Changyu AFIP Global a été ouvert au public en juin 2008. Ce projet de grande envergure a été considéré par Robert Tinlot, ancien président de l'OIV et président d'honneur du château, comme le «premier château viticole global. » D'un coût global d'environ 500 millions yuan, il a été financé par l'entreprise Changyu, mais aussi par les États-Unis, la France, l'Italie et le Portugal ; d'où l'acronyme dans le nom du château (en anglais, AFIP : A comme Amérique ; F comme France; I comme Italie et P comme Portugal).

Globalement, Beijing Château Changyu AFIP Global se présente comme un parc à thème viti-vinicole de grande envergure à la fois par sa taille (environ 500 ha), son caractère multi-fonctionnel, la qualité des constructions, le montant des investissements, le caractère international du projet ainsi que l'importance de la fréquentation touristique (plus de 100000 touristes par an).

Sur un plan matériel, l'organisation territoriale générale du parc à thème viti-vinicole est structurée autour de plusieurs éléments : une grande arche à l'entrée ; un premier château de style gothique servant de point de vente des tickets d'entrée, de boutique à vin et de centre de formation œnologique ; un vaste vignoble traversé par une route et une longue pergola ponctuée de panneaux didactiques relatifs à la vigne et au vin ; un villagehôtel européen majoritairement d'architecture française entièrement reconstitué (AFIP Town, Aifeibao xiaozhen) ; un second château de style gothique comprenant un musée, une cave, des salles de dégustation de vin, un atelier création, des salles de réunion, des équipements de fabrication du vin, une immense esplanade engazonnée.

En tant que territoire, Beijing AFIP s'inscrit dans la catégorie des parcs à thème. Mais ce parc à thème ne vise pas seulement à maximiser le nombre d'entrées et à accroître les dépenses unitaires des touristes par le biais des achats de produits dérivés. Il a pour fonction essentielle de faire vendre le vin. Pour atteindre cet objectif, Beijing AFIP a mis en œuvre une stratégie marketing à la fois puissante et sophistiquée. Le défi apparaît ici d'autant plus difficile que la clientèle chinoise ne connaît pas le vin, voire a un a priori plutôt négatif.

Sur un plan fonctionnel, Beijing Château Changyu AFIP Global représente un parc à thème doté de quatre fonctions principales : fabrication de vin ; tourisme ; formation ; loisirs. Mais, d'un point de vue géosémiotique, il s'agit d'un micro-territoire particulier dont la logique est structurée par le cheminement du client chinois suivant un parcours obligé. Il obéit donc à une linéarité temporelle. Ce cheminement passe ainsi successivement par trois éléments principaux : le premier château, le village européen de type français (appelé AFIP Town : Aifeibao xiaozhen, ou Small Resort Hotels Europe : Ouzhou xiaozhen dujia jiudian) et le second château néogothique. Ces éléments peuvent être considérées comme les étapes d'un parcours initiatique, celui d'une initiation à la culture du vin. Ce parcours n'a pas seulement une fonction récréative, ni même commerciale ; il s'apparente aussi à un voyage dans une autre civilisation, dans 
un Ailleurs exotique. L'organisation générale de Beijing Château Changyu AFIP Global est conçue comme un voyage initiatique, une leçon de choses didactique et une expérience sensorielle. Dans ce parcours initiatique à la culture du vin, véritable processus de transition et de conversion à une boisson, à un mode de boire et à des valeurs totalement différents, l'oenotouriste, véritable héros de conte, passe par plusieurs étapes, voire épreuves, pour obtenir l'objet de sa quête : le vin. Beijing Changyu AFIP Global représente le point d'intersection entre un processus macro-territorial, celui de l'émergence du marché du vin en Chine et un processus d'ordre microterritorial, celui de l'initiation des consommateurs chinois à une boisson culturellement allogène, le vin. Diverses animations (fêtes des vendanges, élection d'une Miss Changyu ... ) ont pour objectif d'éduquer à la culture du vin mais aussi de développer une politique de la marque Changyu. Le premier prix est une bouteille Changyu d'une valeur de 1000 euros.

L'approche géo-sémiotique représente une méthode d'approche pertinente pour comprendre le fonctionnement interne de ce territoire oenotouristique émergent. Elle permet de faire ressortir la spécificité d'un parc à thème viti-vinicole en Chine : pour vendre du vin aux Chinois, il ne suffit pas de déployer une stratégie marketing classique, il est nécessaire de convertir le consommateur chinois à une civilisation différente. Ce parc à thème sur le vin, comme sans doute tout parc à thème sur le vin en Chine, se doit de s'inscrire dans une logique d'interculturalité. Ainsi, l'approche géo-sémiotique permet aussi de comprendre comment la stratégie géomarketing intègre la dimension culturelle pour initier les consommateurs chinois au vin.

En même temps, un tel parc à thème ne peut qu'être victime de son succès. L'efficacité de cette méthode de marketing comporte en elle-même de puissantes contradictions : d'un côté, il fonctionne comme une machine à convertir les clients chinois à la consommation de vin ; de l'autre, cette machine initiatique débouche sur un tourisme de masse entrainant la saturation de ce microterritoire.

\section{4. Élaboration d'une base de données sur les entreprises viti-vinicoles en Chine}

Compte tenu de l'immensité du territoire chinois, mais aussi du nombre élevé et croissant des châteaux vitivinicoles, il est de plus en plus malaisé d'avoir une vision globale du fait vini-vinicole chinois à travers la seule approche qualitative et empirique. Il est donc indispensable d'entreprendre une approche quantitative. G. Giroir a ainsi élaboré une base de données multicritère sur l'ensemble des entreprises vitivinicoles en Chine, soit environ 900. Pour toutes ces entreprises, les informations suivantes ont été recueillies : nom (en chinois) ; localisation (région, ville, adresse postale) ; contact (numéro de téléphone, site Internet) ; date de création ; nom du propriétaire ; principaux produits (vin rouge, vin blanc, vin de glace...) et cépages ; superficie du vignoble; volume de production ; niveau de gamme (haut/moyen/bas) ; contrats avec les viti-agriculteurs ; activité de sous-traitance ; marché principal ; activités extra-productives (hôtellerie, restauration, golf, oenotourisme...) ; nombre de salariés ; statut de l'entreprise (privée, dont par actions, étrangère ; publique ; coopérative ; mixte...). Cette base de données offre un outil d'analyse exceptionnel du fait viti-vinicole chinois. L'exploitation des données permettra d'élaborer un portrait statistique mais aussi cartographique sans équivalent des entreprises viti-vinicoles en Chine, leur hiérarchie, leur mode de fonctionnement, leurs structures de production, la nature de leurs activités productives et extra-productives.

\section{Conclusion}

Tel quel, cet état des lieux dresse un bilan des premières recherches effectuées sur le fait viti-vinicole en Chine dans le champ des sciences humaines et sociales, notamment celles de G. Giroir. Ces résultats de recherche s'avèrent fragmentaires et provisoires. Ils procèdent de plusieurs types d'approches et méthodes. Rétrospectif, cet état des lieux se veut aussi prospectif, voire programmatique : il se présente ainsi également comme une sorte de manifeste ou de programme de développement des recherches futures sur le fait viti-vinicole en Chine.

Le cas de la Chine montre un processus d'émergence viti-vinicole dans un contexte géographique et culturel très particulier. Mais au-delà du cas chinois, il ouvre également des perspectives comparatives sur les vignobles émergents en Asie, actuellement en plein essor : Thaïlande, Vietnam, Birmanie, Indonésie, Inde, etc ...

\section{Références}

[1] G. Giroir, « Vin, mondialisation et civilisation en Chine », Douro. Estudos \& Documentos, Porto (Portugal), VII (13), p. 255-284 (2002)

[2] Zhu Mei, Le vin (葡萄酒), Zhongyang renmin zhengfu qinggong yebu, 128 p. (1954)

[3] Gu Qichang, Cinquante ans d'histoire du vin de la Chine nouvelle (新中国葡萄酒五十年), Tianjin renmin chubanshe, 201 p. (1998)

[4] L. Hannah, Lu Zhi et alii, « Climate, wine, and conservation », Proceedings of the National Academy of Sciences, 110 (17), p. 6907-6912 (2013)

[5] D. Boubals, « Le développement de la viticulture en République populaire de Chine », Le Progrès Agricole et Viticole, Montpellier, $\mathrm{N}^{\circ} 29$, octobre, p. 498 (1985)

[6] A. Löwenstein, Weinbau in der Volksrepublik China. Saarbrücken, Verlag Rita Dadder (1991)

[7] P. Jenster, Yiting Cheng, « Dragon wine : developments in the Chinese wine industry », International Journal of Wine Business Research, 20, p. 244-250 (2006)

[8] G. Giroir « L'entreprise Changyu, acteur majeur de la construction du système vitivinicole émergent en Chine », Cultur. Revista de Cultura e Turismo, Ilheus-Bahia (Brésil) : Universitade Estadual de Santa Cruz (2014) (à paraître)

[9] G. Giroir « Le marché du vin en Chine : entre ivresse et réalités », in François Legouy (coord.) Atlas sur la 
vigne et le vin dans la mondialisation, 2 p., A. Colin (2015) (à paraître)

[10] Li Hua, Li Jiagui, Yang Hecai « Gaige kaifang 30 nian zhongguo putao yu putaojiu chanye fazhan huigu » (Review of Grape and Wine Industry Development in Recent 30 Years of China's
Reforming and Opening-up), Xiandai shipin keji (Modern Food Science and Technology), 25, $\mathrm{N}^{\circ}$ 4, p. 343 (2009) (en chinois)

[11] Hong Guangzhu, Zhongguo nianjiu keji fazhan (A history of wine production in China), Beijing : Zhongguo qinggongye chubanshe (2001) 